\title{
Cisplatin-induced non-convulsive posterior reversible encephalopathy syndrome in a 41-year-old woman with metastatic malignant melanoma
}

\author{
Janja Ocvirk ${ }^{1}$, Marko Boc ${ }^{1}$, Martina Rebersek ${ }^{1}$, Tanja Ros ${ }^{2}$ \\ ${ }^{1}$ Department of Medical Oncology, Institute of Oncology Ljubljana, \\ ${ }^{2}$ Unit of Neurology, Institute of Oncology Ljubljana, Ljubljana, Slovenia
}

\begin{abstract}
Background. Cisplatin, a widely used antineoplastic agent usually induces peripheral neuropathy, but can rarely also complicate with encephalopathy, with or without seizures.

Case report. We report a case of a young patient with metastatic malignant melanoma with signs and symptoms of cisplatin-induced non-convulsive posterior reversible encephalopaty syndrome. Within the days shortly after the first cycle of cisplatin based chemotherapy the patient suffered from nausea, vomitus, headache, severe pain at the site of sub-cutaneous metastases and confusion. She later experienced somnolence, cortical blindness and aphasia, but without epileptic seizures.
\end{abstract}

Conclusions. Cisplatin is an effective chemotherapeutic drug but also very toxic one and physicians using it must also be aware of possible encephalopathy.

Key words: neurotoxicity; cisplatin; posterior reversible encephalopathy syndrome

\section{Introduction}

Cisplatin is an effective and also widely used chemotherapeutic drug; it is part of numerous chemotherapeutic schedules used in the treatment of many solid tumours. Most common side-effects that patients experience are nausea, vomiting, nephrotoxicity, ototoxicity and peripheral neurotoxicity. ${ }^{1,2}$ Central nervous system disorders such as seizures, cortical blind-

Received 27 November 2008

Accepted 6 December 2008

Correspondence to: Janja Ocvirk, MD, PhD, Institute of Oncology Ljubljana, Zaloska 2, Ljubljana. Phone: +386 15879 220; Fax: +386 15879 305; E-mail: jocvirk@onko-i.si ness, aphasia, coma, also hemiparesis are rare, but have already been reported many times, ${ }^{3}$ cortical blindness and seizures being reported first time in 1980's. ${ }^{4}$

\section{Case report}

A 41-year-old female patient with metastatic malignant melanoma was admitted to our hospital because of deterioration of her mental and physical state after the first cycle of the second line chemotherapy (ChT) in combination with cisplatin.

Primary malignant melanoma on the left lumbal part of the back was surgically removed in September 2005 (Clark IV, 
Breslow 1.68, ulceration $2 \mathrm{~mm}$, with signs of regression, without microsatelites, without vascular invasion, TNM stage $\mathrm{T} 2 \mathrm{bN} 1 \mathrm{a}(1 /$ 14)M0, stage IIIB). The sentinel node biopsy presented with a $2.5 \mathrm{~mm}$ wide metastasis; therefore the dissection of axilliary lymph nodes was performed.

From February 2006 to May 2007 she was receiving interferon in an adjuvant setting. During the adjuvant treatment there was clinically a locoregional relapse with a $12 \mathrm{~cm}$ bulky mass on the site of the primary tumour, further examination (CT, RTG, and UZ) has shown the dissemination in the lungs and pleura with multiple lung metastases.

She started her first line ChT with dakarabazine-DTIC (1000 mg/m² i.v., day 1, 21 days) in May 2007. After two cycles the further progression of the disease was observed, locoregionally and systemically. The bulky mass on the left lumbal part of her back was now clinically $15 \mathrm{~cm}$ in diameter and was at least $3 \mathrm{~cm}$ above skin level.

The second line treatment was started in June 2007, she received the first cycle of ChT with cisplatin $\left(80 \mathrm{mg} / \mathrm{m}^{2}\right.$ i.v., day 2., 21 days), lomustin-CCNU ( $80 \mathrm{mg} / \mathrm{m}^{2}$ p.o., day 1., 21 days) and vinblastin $\left(3 \mathrm{mg} / \mathrm{m}^{2} 24 \mathrm{~h}\right.$ infusion, day 1, 21 days) with premedication with dexamethason $20 \mathrm{mg}$ i.v., granisetron $3 \mathrm{mg}$ i.v., manitol $500 \mathrm{ml}$ i.v. and $\mathrm{MgSO}_{4}$ $5 \mathrm{ml}$ i.v.. She was also hydrated with 3000 $\mathrm{ml}$ of $0.9 \% \mathrm{NaCl}$. She ended her first cycle without acute toxic event.

Shortly after she got home, she started suffering from nausea, vomitus, headache, severe pain at the site of subcutaneous mass and confusion. On the day of the admission she deteriorated even more, followed by somnolence, cortical blindness and aphasia, but without epileptic seizures. At home she was continuing with buprenorfin transdermal patch (Transtec ${ }^{\circledR}$ ) $35 \mu \mathrm{g}$, short acting oral morphine (Sevredol ${ }^{\circledR}$ ) 10 mg for breaking through pain, diclofenak tbl. (Olfen $\mathrm{SR}^{\circledR}$ ) $100 \mathrm{mg} /$ day, pregabalin tbl. (Lyrica ${ }^{\circledR}$ ) $75 \mathrm{mg} /$ day, pantoprazol tbl. $\left(\right.$ Controloc $\left.^{\circledR}\right) 1$ tbl/day, metoklopramid tbl. $\left(\right.$ Reglan ${ }^{\circledR}$ ) 3x1 tbl/day in granisetron tbl. $\left(\right.$ Kytril $\left.^{\circledR}\right) 2$ mg when needed.

WHO performance status on admission was 3-4, the patient was affected, she was in pain, aphasic, somnolent, almost comatose. Blood pressure was $110 / 70 \mathrm{mmHg}$, heart rate was $80 / \mathrm{min}$, she was afebrile. Meningeal signs were negative, her breathing and heart signs were normal. Abdomen was soft, without pathologic mass or liver palpable, without tenderness. The bulky subcutaneous mass on the site of primary tumour was smaller, only about $10 \mathrm{~cm}$ in diameter and has flattened.

The laboratory report has shown mild hypokaliemia (3.7) and hyponatriemia (132), without any signs of dehydration, with elevated WBC (14.9) and CRP (39). The laboratory report did not explain the patient's state; electrolytes, magnesium, calcium were within limits, her blood sugar level was normal.

\section{Differential diagnosis}

At first we suspected that she developed metastasis in the CNS, as CNS is frequent site of dissemination in malignant melanoma. She was presented with possible signs of increased intracranial pressure with severe headache and vomitus, confusion, aphasia, somnolence and later stupor. So, she was started on antioedematic therapy with corticosteroids and manitol solution, but her state was improving slower than expected in increased intracranial pressure.

To exclude the possibility of morfine intoxication, morphine therapy was discontinuated.

Urgent computer tomography (CT) of her brain did not show any proof of intracranial dissemination; bedsides, the neurologic exam showed that the patient was deeply somnolent, without speech contact, though 


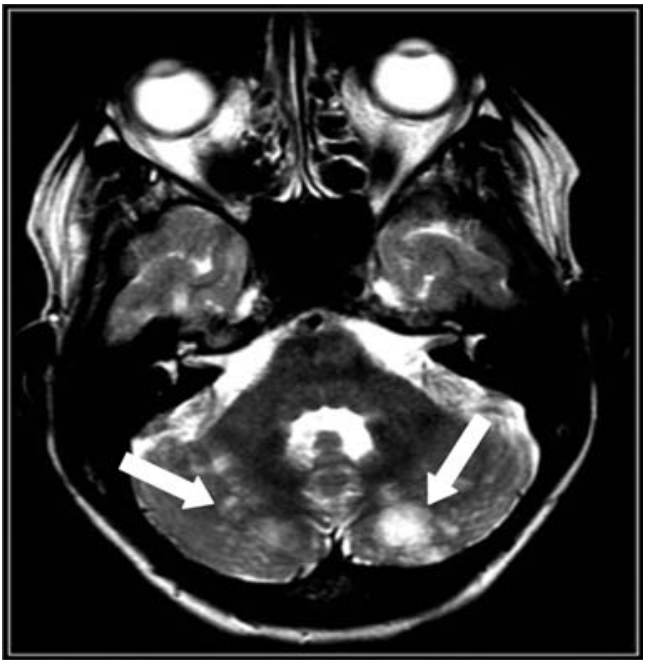

Figure 1a. Oedema in the cerebellum (T2, without contrast).

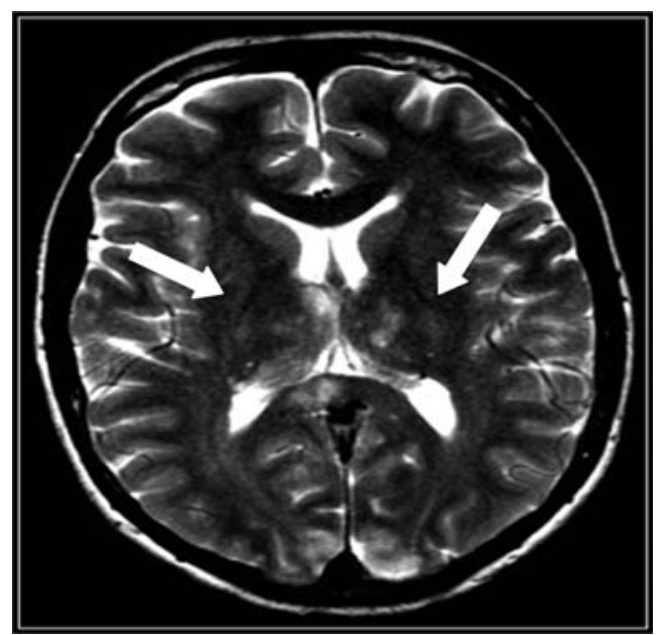

Figure 1c. Oedema in the corpus callosum (T2, without contrast).

she did react on a call with movements and restlessness, but she did not open her eyes. There were neither focal signs of central nervous damage nor signs of pyramidal tract lesion; meningeal signs were negative. The lumbar punction was performed; cerebrospinal fluid was entirely normal, without malignant cells and with negative tests on bacterial and viral examination.

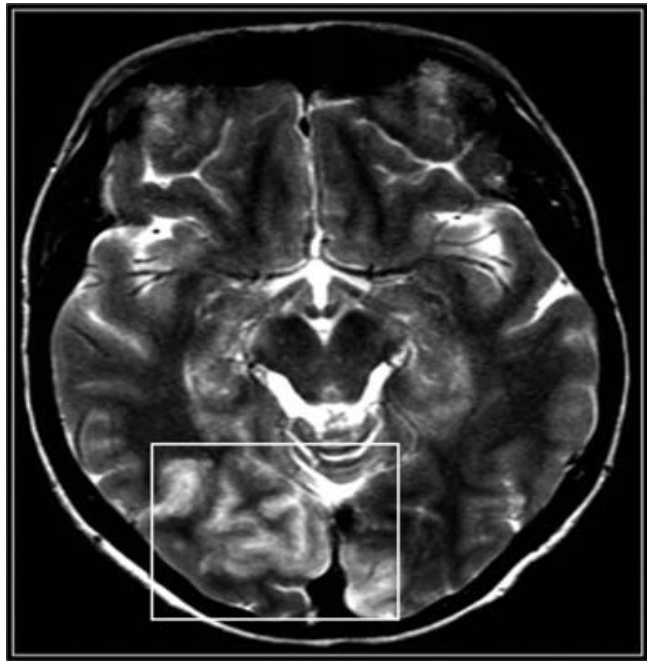

Figure 1b. Oedema in the occipital cortex (T2, without contrast).

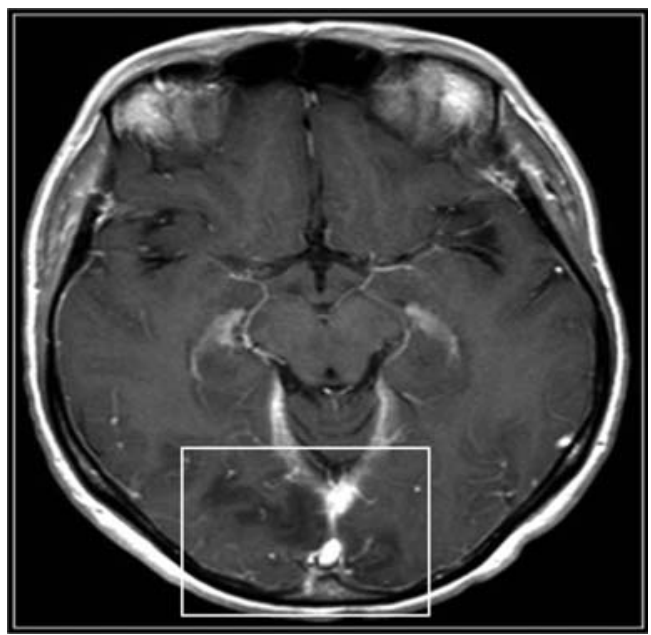

Figure 1d. Occipital cortex (T2, with contrast).

\section{MRI presentation}

At that time MRI of her brain was performed. She had typical neuroradiological changes in the form of oedema in supraand infra-tentorial areas with hyperintensity on T2-weighted MRI imaging, seen in the occipital cortex, cerebellum, basal ganglia and corpus callosum (Figures 1a, 1b, 1c, 1d). 


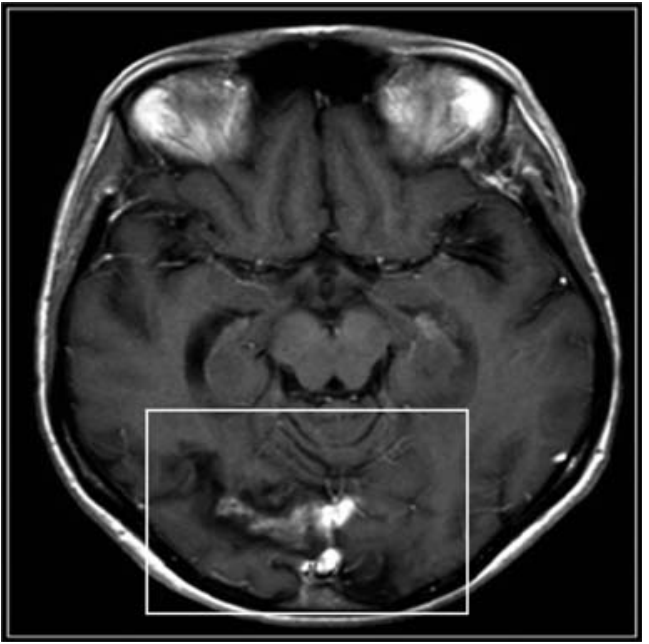

Figure 3. Control MRI-occipital cortex (T1, with contrast), infarction on the site of previous oedema.

\section{EEG presentation}

Electroencephalogram showed diffuse slow-wave activity with frequent generalized paroxysms of sharp waves and slow delta activity (1.5-2 Hz) (Figure 2), EEG findings were compatible with reversible non-convulsive encephalopathy.

\section{Further course of disease}

During hospitalization we continued with the symptomatic treatment, her condition slowly improved, on the third and fourth day she was more alert, but we disclosed blindness, she didn't complain about. The ophthalmologic examination at bedside was normal, except the possibility to test vision and visual fields. Her vision and other symptoms slowly improved and so did MRI (Figure 3) and EEG findings (Figure 4). Repeated MRI disclosed an ischemic lesion in the occipital cortex, otherwise rarely seen complication of PRES, with partly completed regression of other changes. After fifteen days she was discharged from the hospital, fully conscious, with much improved vision, and without dysphasia.

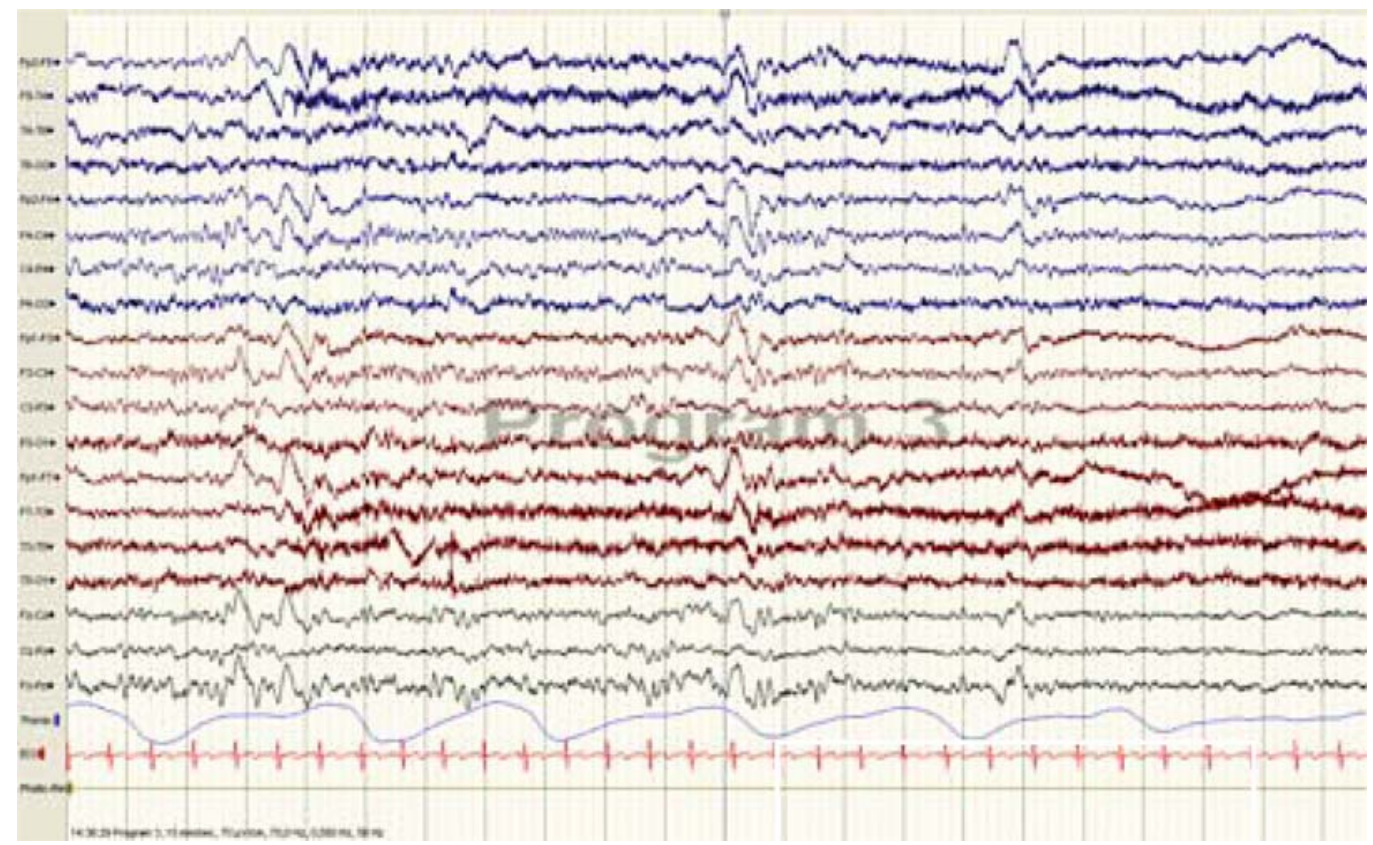

Figure 2. EEG two weeks after admission with diffuse slow wave activity and generalised paroxysms of sharp waves and slow delta activity. 
She received next two cycles of ChT with cisplatin after twelve days and the third one later on, both times in split doses. No central neurotoxicity reoccurred, but after the malignant disease progressed, ChT was stopped and the patient died three and a half months after starting cisplatin-based ChT.

\section{Discussion}

Cisplatin side effects are often predictable in terms of their onset and duration, being most common nausea, vomitus, kidney toxicity and peripheral neuropathy with numbness, paresthesias, and occasionally pain, which usually begins in the toes and fingers, spreading proximally to affect the legs and arms. ${ }^{5}$ In the presented patient, clinical signs and symptoms, MRI and EEG findings were compatible with rare central nervous toxicity of cisplatin-induced non- convulsive posterior reversible encephalopathy syndrome that occurred after the first dose of cisplatin-based ChT. The causal relationship to the agent was made after excluding other causes of the condition, such as progression of malignant disease, metabolic, iatrogenic, infectious and vascular causes. As in other cerebral diseases the MRI was essential in differential diagnosis, ${ }^{6}$ but we found the main reason for the neurological disorder with contextual understanding of other clinical findings.

Of the other concurrent medications that are potentially central neurotoxic she also received lomustine, but according to data that we found, nitrosoureas are associated with little neurotoxicity at conventional doses, toxic effect is possible after cumulative and high iv doses, 7,8 which is not the case in our patient.

According to published data the exact mechanism for the emergence of cisplatin-induced encephalopathy is not known,

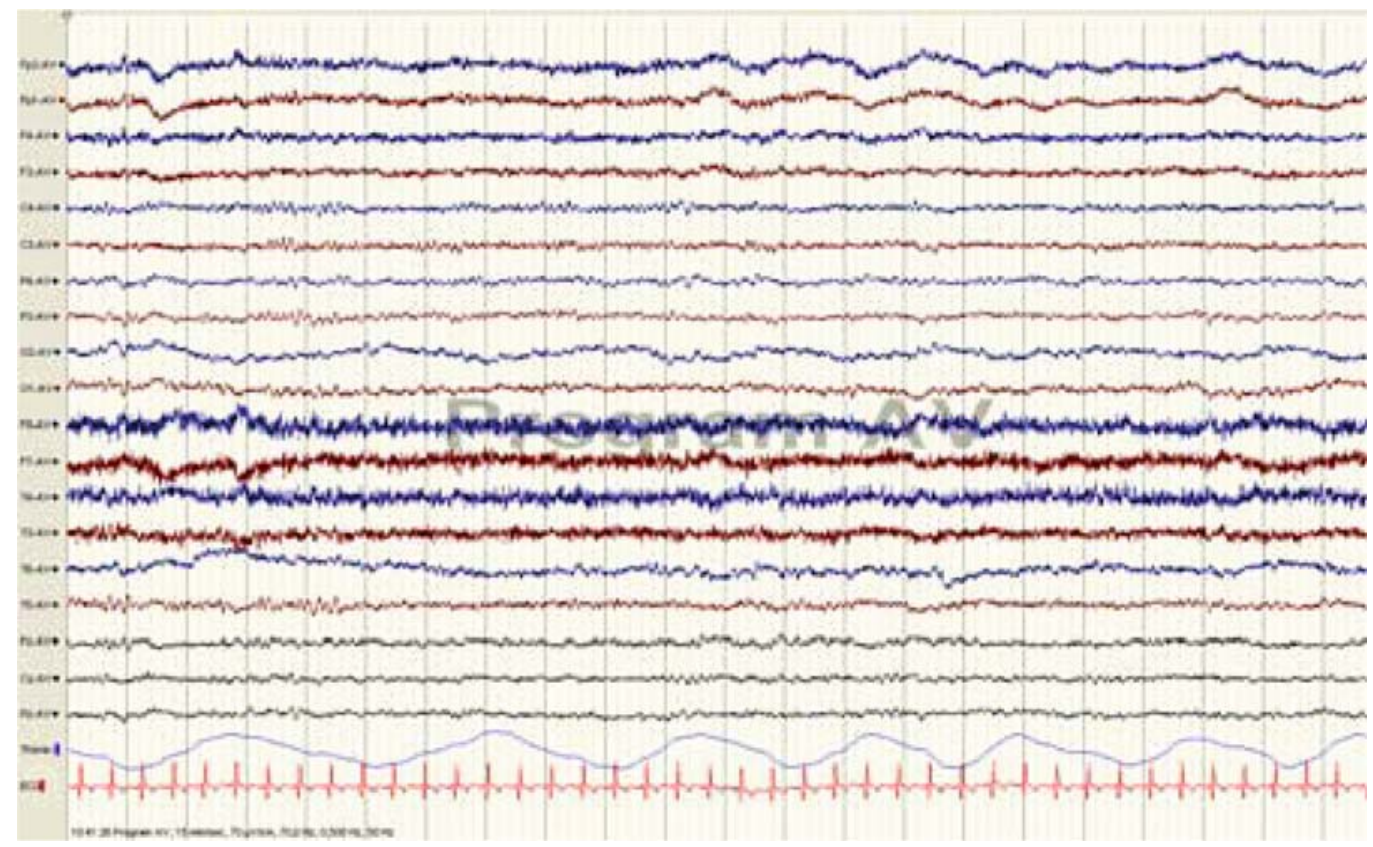

Figure 4. Control EEG after three months indicating improvement with disappearance of sharp and slow wave delta activity. 
one hypothesis is that it may be caused by vascular events, other is that mechanism may be toxic encephalopathy, e.g. heavy metal toxicity and demyelinization. ${ }^{9}$ Fever, thrombocytopenia, neutropenia, renal insufficiency, metabolic disturbances also contribute to CNS toxicity of cisplatin, but that was not the case in our patient. Neurotoxicity typically starts within two weeks after the treatment with cisplatin, with or without seizures, accompanied by acute or sub acute confusional state, central visual problems (even blindness) and often headache. ${ }^{10}$ Our patient suffered from nausea, vomitus, headache, severe pain at the site of sub-cutaneous metastases and confusion, she later experienced somnolence, cortical blindness and aphasia, but without epileptic seizures, a combination characteristic of a less common, non-convulsive encephalopathy. Her symptoms resolved, she received the treatment with another two cycles, both times with split doses of cisplatin, decided by intending medical oncologist according to good clinical practice for minimizing the side effects. Both cycles were administered according to the same schedule and adverse events did not repeat. On control MRI, an ischemic lesion, a rarely seen complication to be present also at autopsy of such patients, was found.

\section{Conclusions}

According to published cases, cisplatin-induced central nervous toxicity is a relatively rare complication in cancer patients, but with the widespread use of cisplatin this rare disorder should be considered, especially if neurologic symptoms as described in this article occur. When diagnosed at an early stage it is usually a reversible condition, which requires adequate treatment of seizures, symptomatic treatment and withdrawal or cautiousness with cisplatin therapy. ${ }^{11}$ It is important do differentiate neurotoxicity of cisplatin from signs of tumour progression. ${ }^{12}$

\section{References}

1. New PZ. Neurological complications of chemotherapeutic and biological agents. Continuum: lifelong learning in neurology. Neuro-Oncol 2005; 11(5):116-152.

2. Kaplan RS, Wiernik PH. Neurotoxicity of antineoplastic drugs. Sem Oncol 1982; 9: 103-30.

3. Lyass O, Lossos A, Hubert A, Gips M, Peretz T. Cisplatin-induced non-convulzive encephalopathy. Anticancer Drugs 1998; 9: 100-4.

4. Berman IJ, Mann MP. Seizures and transient cortical blindness associated with cis-platinum (II) diamminedichloride (PDD) therapy in a thirty year old man. Cancer 1980; 45: 764-66.

5. UpToDate online, version 16.3. Neurologic complications of platinum-based chemotherapy. Available: http://www/uptodateonline.com/. 30 October, 2008.

6. Franko A, Holjar-Erlić I, Miletić D. Lateral ventricle epidermoid. Radiol Oncol 2008; 42: 66-8.

7. Burger PC, Kamenar E, Schold SC, Fay JW, Phillips GL, Herzig GP. Encephalomyelopathy following high-dose BCNU therapy. Cancer 1981; 48: 131837.

8. Posner JB. Neurologic complication of cancer. Philadelphia: FA Davis; 1995. p. 282-310.

9. Connolly RM, Doherty CP, Beddy P, O'Byrne K. Chemotherapy induced reversible posterior leucoencephalopathy syndrom. Lung Cancer 2007; 56: 459-63.

10. Steeghs N, de Jongh E, Sillevis Smith PAE, van den Bent MJ. Cisplatin-induced encephalopathy and seizures. Anticancer Drugs 2003; 14: 443-6.

11. Ros T, Rebersek M, Boc M, Ocvirk J, Jereb S, Dolenc-Groselj I, et al. Cisplatin induced encephalopathy. [Abstract]. EANO 2008: P152. NeuroOncol 2008; 10(6): 1119.

12. Verschraegen C, Conrad CA, Hong WK. Subacute encephalopathic toxicity of cisplatin. Lung Cancer 1995; 13: 305-9. 